\title{
On the Existence of Designer Magnetic Superatoms
}

\author{
Xinxing Zhang, ${ }^{\dagger}$ Yi Wang, ${ }^{\dagger}$ Haopeng Wang, ${ }^{\dagger}$ Alane Lim, ${ }^{\dagger}$ Gerd Gantefoer, ${ }^{\ddagger}$ Kit H. Bowen, ${ }^{*}{ }^{\dagger}$ \\ J. Ulises Reveles, ${ }^{\S}$ and Shiv N. Khanna*,§ \\ ${ }^{\dagger}$ Department of Chemistry, Johns Hopkins University, Baltimore, Maryland 21218, United States \\ ${ }^{*}$ Department of Physics, Universitaet Konstanz, Konstanz, Germany \\ ${ }^{\S}$ Department of Physics, Virginia Commonwealth University, Richmond, Virginia 23284, United States
}

Supporting Information

\begin{abstract}
The quantum states in small, compact metal clusters are bunched into electronic shells with electronic orbitals resembling those in atoms, enabling classification of stable clusters as superatoms. The filling of superatomic orbitals, however, does not generally follow Hund's rule, and it has been proposed that magnetic superatoms can be stabilized by doping simple metal clusters with magnetic atoms. Here, we present evidence of the existence of a magnetic superatom and the determination of its spin moment. Our approach combines first principles studies with negative ion photoelectron experiments and enables a unique identification of the ground state and spin multiplicity. The studies indicate $\mathrm{VNa}_{8}$ to be a magnetic superatom with a filled d-subshell and a magnetic moment of $5.0 \mu_{\mathrm{B}}$. Its low electron affinity is consistent with filled subshell and enhanced stability. The synthesis of this species opens the pathway to investigate the spin-dependent electronics of the new magnetic motifs.

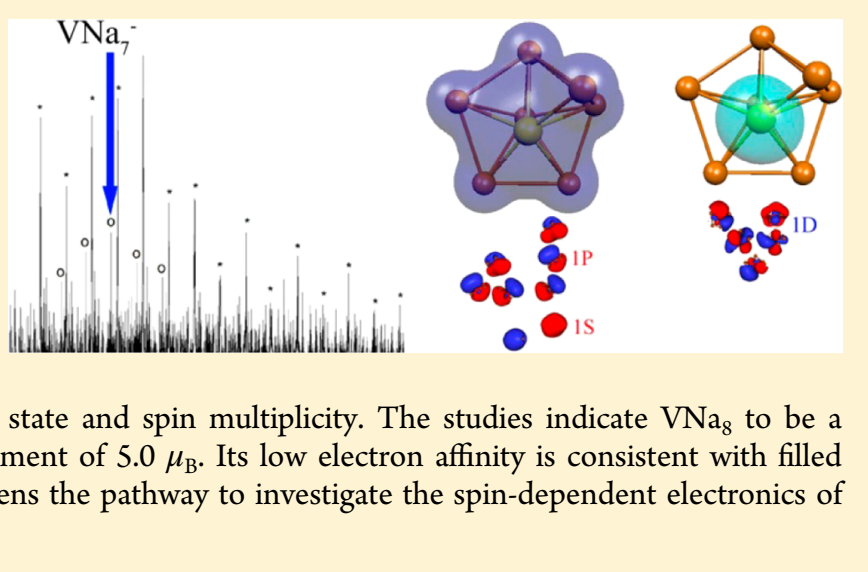

\section{INTRODUCTION}

There is now overwhelming evidence that the electronic states in small, compact metal clusters are grouped into shells and that the orbital shapes of the states in a shell resemble the situation in atoms. ${ }^{1-17}$ This similarity has led to the conceptual development permitting clusters to be classified as superatoms. The electronic shells in superatoms can be labeled by examining the nodal shapes of their molecular orbitals, and these roughly order as $1 \mathrm{~S}, 1 \mathrm{P}, 1 \mathrm{D}, 2 \mathrm{~S}, 1 \mathrm{~F}, 2 \mathrm{P}, \ldots$, where the uppercase letters are used to distinguish them from atomic orbitals that are generally labeled by lowercase symbols. Each orbital in a superatom (superorbital) is generally distributed over multiple atoms, and the filling of orbitals in a given shell does not generally follow Hund's rule of maximum spin multiplicity. Any attempt to fill degenerate/closely spaced states by unpaired electrons is counteracted by spatial distortions that can break the electronic degeneracy and lower the total energy with states occupied with paired electrons (Jahn-Teller effect). ${ }^{18}$ This accounts for the fact that simple metal clusters like $\mathrm{Na}_{x}$ generally have their lowest spin multiplicities in their ground states, as the gain in energy associated with Jahn-Teller distortion is greater than that due to Hund's coupling. ${ }^{18}$ One way to stabilize a magnetic superatom is to introduce a transition metal atom with partially filled d-states into a simple metal cluster. It is then possible to stabilize magnetic configurations for D-states, as the superatomic D-states result from the hybridization with atomic d-states.

We recently suggested that a $\mathrm{VNa}_{8}$ cluster may offer such a combination. ${ }^{17} \mathrm{~A} \mathrm{~V}$ atom has a $3 \mathrm{~d}^{3} 4 \mathrm{~s}^{2}$ valence configuration, while a $\mathrm{Na}$ atom is an alkali atom with $3 \mathrm{~s}^{1}$ valence state. $\mathrm{A} \mathrm{VNa}_{8}$ cluster has 13 valence electrons, and the ground-state geometry is a square antiprism of $\mathrm{Na}$ atoms with an interior $\mathrm{V}$ atom. The electronic ground state of the cluster has a $1 S^{2} 1 P^{6} 1 D^{5}$ distribution of electrons. Since the $1 \mathrm{D}$-state has a strong component from the atomic $\mathrm{d}$-state of $\mathrm{V}$, it undergoes a large exchange splitting, leading to a cluster with a spin magnetic moment of $5.0 \mu_{\mathrm{B}} \cdot{ }^{17}$ Until now, there has been no experimental evidence for the formation of such magnetic states and the nature of the associated ground state.

In this work we provide evidence for the existence of such stable clusters as well as a characterization of their magnetic states. The ground states of atoms or molecules can be generally probed through spectroscopic techniques. However, such assignments for clusters having a finite number of atoms are difficult. The measurement of the magnetic moment at small cluster sizes is also a difficult task due to experimental limitations. This is because such measurements for small clusters are usually carried out using the Stern Gerlach setup: ${ }^{19}$ a beam of clusters pass through a gradient magnet that orients the magnetic moments in the direction of the field while the gradient deflects the beam. The reduction in size reduces the magnetic anisotropy, and small clusters are known to undergo superparamagnetic relaxations. ${ }^{20}$ Consequently, the net magnetization and the deflections for clusters having moments of only a few Bohr magneton are small and at the limit of the

Received: January 24, 2013

Published: March 4, 2013 
accuracy of the experiments. An alternate choice, used here, is to combine negative ion photoelectron spectroscopy with firstprinciples calculations. In these experiments, mass-selected cluster anions are subjected to laser irradiation where the photon detaches the excess electron. ${ }^{21,22}$ The process involves a transition from the ground state of the anion to the ground and excited states of the neutral, and a measurement of the energy of the resulting electron allows the photoelectron spectra to provide a fingerprint of the electronic structure of the corresponding neutral clusters. Such measurements can be compared with theoretical calculations via the transition energies. A match between the calculated transitions and the experiments provides an indirect determination of the multiplicity and the spin magnetic moment of the cluster. Previous studies have indicated that such a procedure is quite accurate and can even distinguish between isomers with close-lying energies. $^{23}$

\section{EXPERIMENTAL AND COMPUTATIONAL METHODS}

Anion photoelectron spectroscopy was conducted by crossing a massselected negative ion beam with a fixed energy photon beam and analyzing the energies of the resultant photodetached electrons. This technique is governed by the well-known energy-conserving relationship, $h \nu=\mathrm{EBE}+\mathrm{EKE}$, where $h \nu, \mathrm{EBE}$, and EKE are the photon energy, electron binding energy (transition energy), and the electron kinetic energy, respectively. The details of our apparatus have been described elsewhere. ${ }^{24,25}$ Briefly, the photoelectron spectra were collected on an apparatus consisting of an ion source, a linear timeof-flight mass spectrometer for mass analysis and selection, and a magnetic-bottle photoelectron spectrometer for electron energy analysis (resolution $\sim 35 \mathrm{meV}$ at $1 \mathrm{eV}$ EKE). The third harmonic $(355 \mathrm{~nm}, 3.49 \mathrm{eV} /$ photon) of a Nd:YAG was used to photodetach electrons from the cluster anion of interest. Photoelectron spectra were calibrated against the well-known atomic lines of the copper anion. ${ }^{26}$

The $\mathrm{V}_{1,2} \mathrm{Na}_{n}{ }^{-}$clusters were generated using a pulsed arc cluster ionization source (PACIS), which has been described in detail elsewhere. ${ }^{27}$ In brief, a $\sim 20 \mu$ s long $150 \mathrm{~V}$ electrical pulse applied across anode and sample cathode of the discharging chamber vaporizes the vanadium and sodium atoms. The sample cathode was prepared in a nitrogen protected glovebox, where vanadium powder and fresh sodium were mixed and firmly pressed onto a copper rod. About 10 bar of ultrahigh purity helium gas then flushed the vanadium-sodium plasma mix down in a $20 \mathrm{~cm}$ flow tube, where it reacted, formed clusters, and cooled. Anions generated by this method were then massselected for photoelectron spectroscopic studies.

First principles electronic structure calculations on neutral and anionic $\mathrm{V}_{1,2} \mathrm{Na}_{n}{ }^{-}$clusters were performed within the generalized gradient density functional theory (DFT) formalism. The calculations were carried out using the linear combination of Gaussian-type orbitals centered at the atomic positions, employing the DFT Kohn-Sham code deMon $2 \mathrm{k}^{28,29}$ The exchange and correlation effects were incorporated through the functional proposed by Perdew et al. ${ }^{30} \mathrm{~A}$ variational fitting of the Coulomb potential was employed in order to avoid the calculation of four-center electron repulsion integrals. ${ }^{31}$ The exchange-correlation potential was calculated via a numerical integration from the orbital density. All electrons were treated explicitly using the double- $\zeta$ valence plus polarization (DZVP) basis sets. ${ }^{32}$ The auxiliary density was expanded in primitive Hermite Gaussian functions using the GEN-A2 auxiliary function set. For each cluster size, the ground-state configuration was obtained by starting from several initial configurations and spin states and optimizing the geometries without symmetry constrains in delocalized internal coordinates. ${ }^{33} \mathrm{~A}$ frequency analysis was carried out to ascertain the stability of the ground state. The time-dependent(TD) DFT calculations were performed for the neutral clusters at the anionic's optimized geometries. ${ }^{34}$ Given an anionic cluster of multiplicity $\mathrm{m}$, we calculated the total energies and excitation energies of the corresponding neutral cluster at $m+1$ and $m-1$ multiplicities, as the photodetached electron could be taken either from the majority or the minority levels. Then, we added the total and the excitation energies to get the total energies of the neutral excited states. Next, we calculated the vertical detachment energies (VDE) as the energy difference between the anionic cluster and all the calculated neutral states. The energy differences were further broadened by Gaussians of width 0.02 $\mathrm{eV}$ and the two spectra of the $m+1$ and $m-1$ multiplicities were added to generate the theoretical spectra shown in Figures 2, 3 and S1.

\section{RESULS AND DISCUSSION}

Figure 1 shows the mass spectrum of the clusters generated in beams. Two series of clusters were generated. The $\mathrm{VNa}_{x}{ }^{-}$series

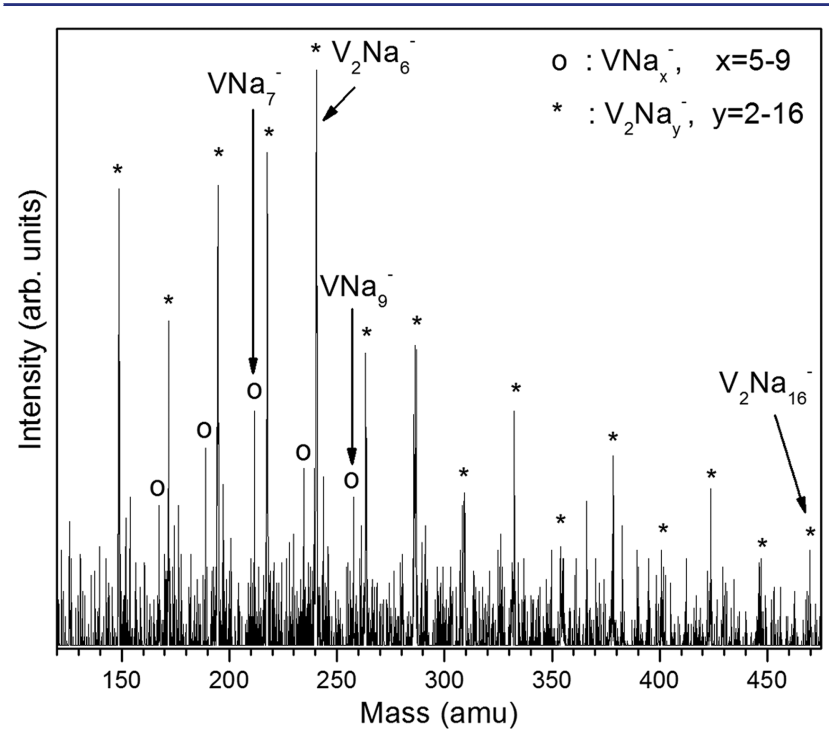

Figure 1. Time-of-flight mass spectrum of bimetallic $\mathrm{V}_{1-2} \mathrm{Na}_{n}{ }^{-}$cluster anions.

corresponds to a single $\mathrm{V}$ atom, while the $\mathrm{V}_{2} \mathrm{Na}_{y}{ }^{-}$series corresponds to $2 \mathrm{~V}$ atoms. The $\mathrm{VNa}_{x}{ }^{-}$series, identified by hollow circles, is marked with a peak at $\mathrm{VNa}_{7}^{-}$, while the $\mathrm{V}_{2} \mathrm{Na}_{y}{ }^{-}$series, identified by asterisks, is peaked around $\mathrm{V}_{2} \mathrm{Na}_{6}{ }^{-}$ with a significant drop in intensity thereafter. Both of the series show a peak at a cluster size containing eight atoms. The variations in the intensity in mass spectra are generally attributable to the experimental conditions. As we are primarily interested in the magnetic character and since our previous theoretical studies had identified $\mathrm{VNa}_{8}$ to be a magnetic superatom, ${ }^{17}$ the negative ion photoelectron spectra were collected for the $\mathrm{VNa}_{x}{ }^{-}$clusters containing 6, 7, 8, and $9 \mathrm{Na}$ atoms. These are shown in Figures 2 and 3.

Among the quantities of interest in a particular system are its adiabatic electron affinity (AEA) and the first and second peak center locations which identify the VDE's from the anion, with a spin multiplicity of $\mathrm{m}$, to its corresponding neutral cluster with the same geometry as the anion and with multiplicity $m$ \pm 1 . As the AEA corresponds to a transition from the ground state of the anion to the relaxed configuration of its neutral counterpart, the experimental determination of its value is often based on the onset of the first peak in the observed spectra. On the other hand, the vertical transitions representing the vertical transition from the anion to the neutral at the anion's geometry are more clearly defined. These transitions are sensitive to the geometry and the multiplicity of the anion's ground state and hence provide a reliable fingerprint of the electronic state. In 

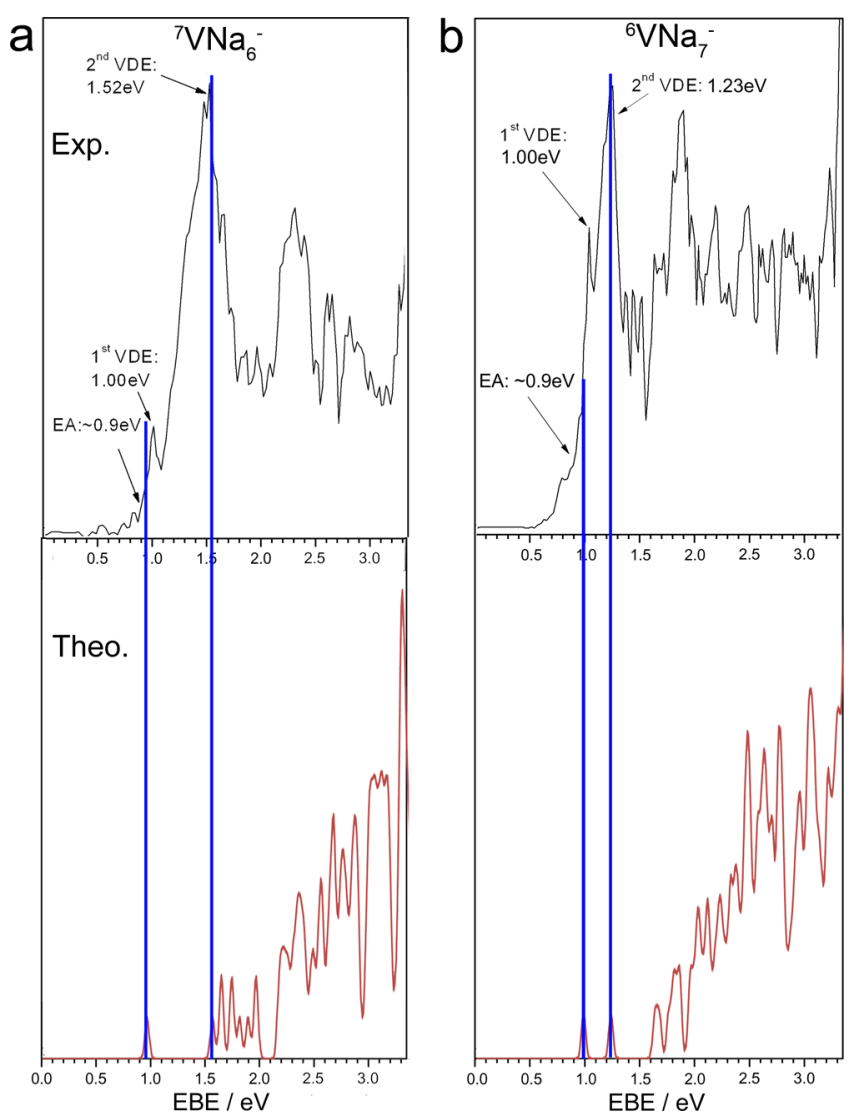

Figure 2. Photoelectron spectra at $355 \mathrm{~nm}$ for $\mathrm{VNa}_{x}{ }^{-}$clusters $(n=6-$ 7). The calculated absorption spectra of neutral $\mathrm{VNa}_{8}$ is marked in red. The calculated vertical detachment energies are marked with blue lines.

fact, we had earlier used them to distinguish between isomers of a $\mathrm{Fe}_{3}{ }^{-}$cluster and to provide an accurate value for the spin magnetic moment of this and other clusters. ${ }^{23}$

Experiments were also undertaken on $\mathrm{V}_{2} \mathrm{Na}_{y}{ }^{-}$clusters containing 6 and $13-16 \mathrm{Na}$ atoms. $\mathrm{V}_{2} \mathrm{Na}_{6}{ }^{-}$corresponds to a peak in the mass spectrum of $\mathrm{V}_{2} \mathrm{Na}_{y}{ }^{-}$species. These obtained photoelectron spectra are given in Figure S1. Figure 4 shows the calculated ground states of the neutral and anionic $\mathrm{VNa}_{x}{ }^{-}$ clusters containing $6-12 \mathrm{Na}$ atoms. $\mathrm{VNa}_{6}{ }^{-}$is marked by two isomers with different spin multiplicities. The ground state of the neutral resembles one of the anionic isomers. The photoelectron spectrum can be used to identify if both isomers are present in the anion beam. The ground state of $\mathrm{VNa}_{7}{ }^{-}$has an exposed $\mathrm{V}$ atom and the neutral cluster and anion have similar geometries. $\mathrm{VNa}_{8}{ }^{-}$is the smallest cluster which has an endohedral $\mathrm{V}$ atom enclosed within a square antiprism of $\mathrm{Na}$ atoms. The cluster has a high spin multiplicity of $7 . \mathrm{VNa}_{9}{ }^{-}$is obtained from $\mathrm{VNa}_{8}{ }^{-}$by adding a capping $\mathrm{Na}$ atom on the upper square face with a spin multiplicity of 6. Similarly, $\mathrm{VNa}_{10}{ }^{-}$is obtained from $\mathrm{VNa}_{9}{ }^{-}$by adding a capping $\mathrm{Na}$ atom on the upper square face, and with a reduced spin multiplicity of 5. The decrease in spin multiplicity continues as further $\mathrm{Na}$ atoms are added. $\mathrm{VNa}_{11}{ }^{-}$presents an incomplete icosahedral structure and $\mathrm{VNa}_{12}{ }^{-}$an icosahedral structure with spin multiplicity of 4 and 3, respectively.

The ground states of $\mathrm{V}_{2} \mathrm{Na}_{y}{ }^{-}(y=4-16)$ are shown in Figure S2. In all of these clusters the ground state corresponds to a dimer of $\mathrm{V}$ atoms surrounded by $\mathrm{Na}$ atoms. These clusters have the lowest spin multiplicities among the systems studied herein
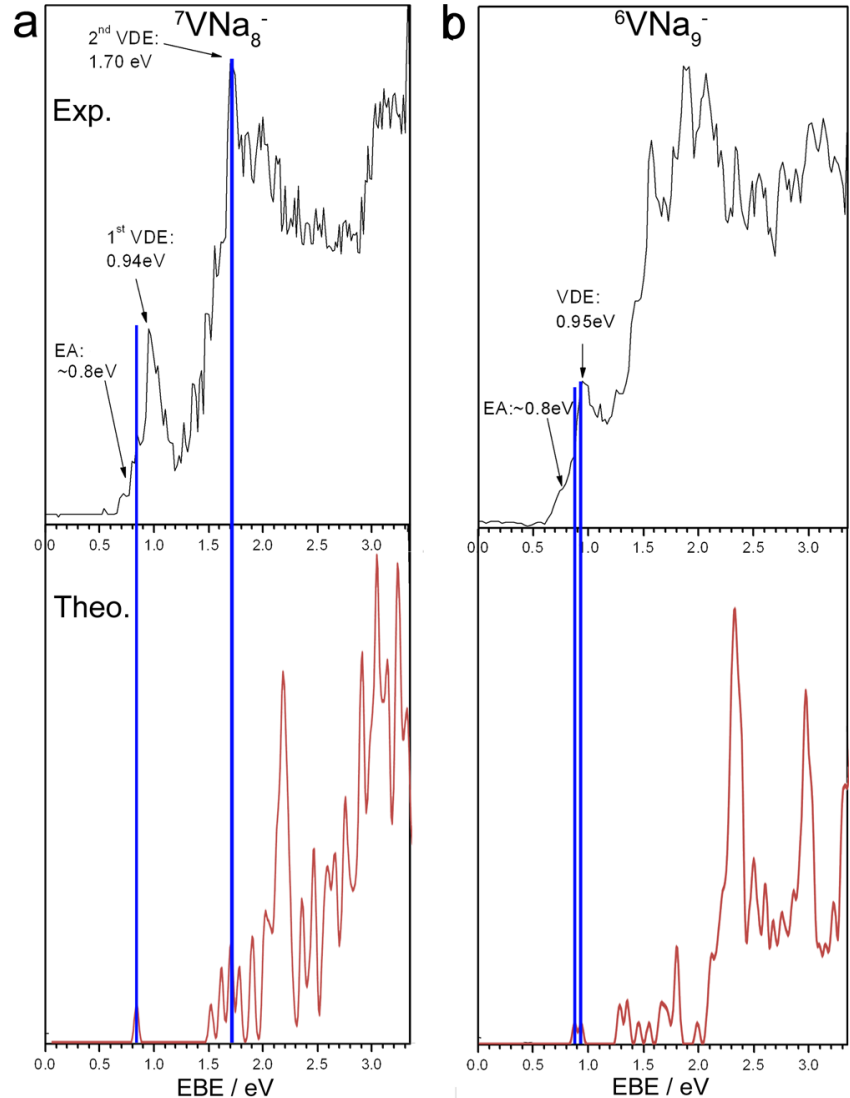

Figure 3. Photoelectron spectra at $355 \mathrm{~nm}$ for $\mathrm{VNa}_{x}{ }^{-}$clusters $(n=8-$ 9).

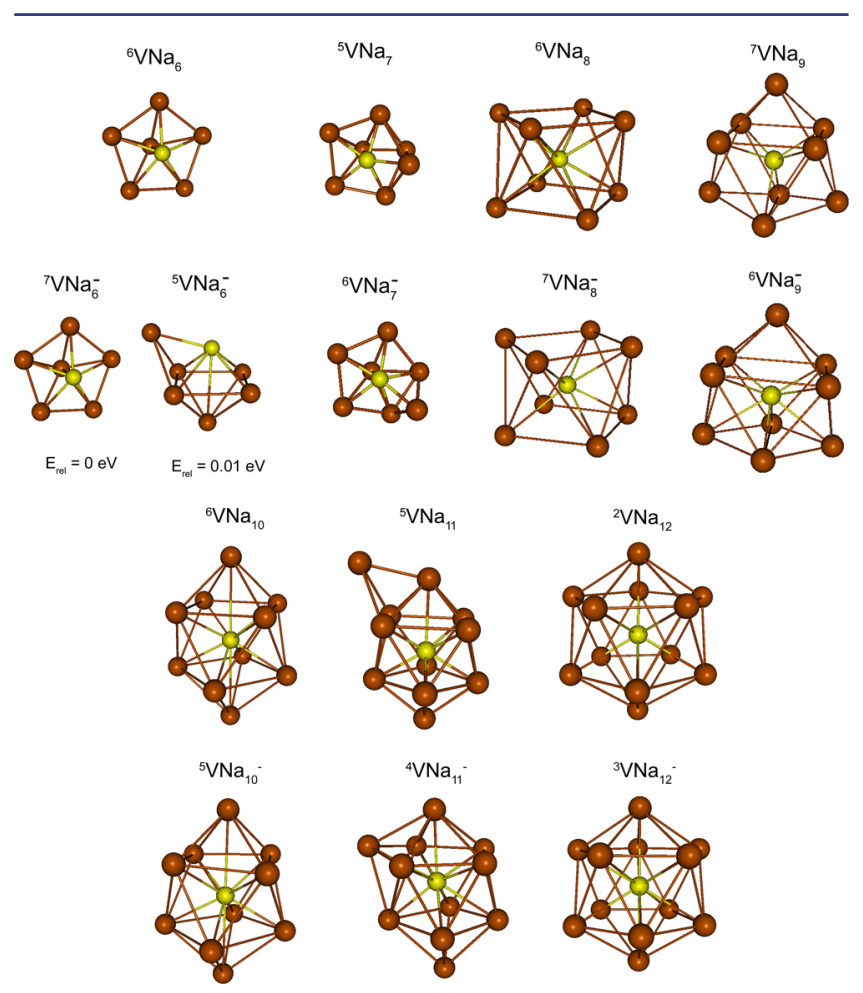

Figure 4. Ground-state geometries of neutral and anionic $\mathrm{VNa}_{x}(n=$ 4-12) clusters The superscripts indicate spin multiplicity. Brown and yellow circles represent sodium and vanadium atoms, respectively. 
(singlet and doublet for even and odd electron systems, respectively), except for $\mathrm{V}_{2} \mathrm{Na}_{6}$ which has a triplet ground state. As the present article is focused on magnetic species, we will primarily concentrate on single $\mathrm{VNa}_{x}{ }^{-}$clusters.

A comparison between the photoelectron spectra and the theoretical calculations is the key to determining the ground states of these clusters. The calculated ground states of the anions and neutrals were used to calculate AEA values. We also determined VDE values from the anions of multiplicity $m$ to their neutral clusters of multiplicity, $m \pm 1$ by starting from the ground state of the anion and calculating the energy of the neutral species at the geometry of the anion, i.e., by removing an electron from the majority or minority states. The VDE's correspond to peaks in the photoelectron spectrum. Table 1 shows the calculated values for VDE corresponding to $m \pm 1$ and the AEA for $\mathrm{VNa}_{x}{ }^{-}$clusters containing 5-9 $\mathrm{Na}$ atoms, and all the experimentally determined transitions.

Table 1. Experimental and Theoretical VDE and AEA Values (eV) along with Calculated H-L Gaps (eV) for Anionic $\mathrm{V}_{1-2} \mathrm{Na}_{y}{ }^{-}$Clusters

\begin{tabular}{|c|c|c|c|c|c|c|c|}
\hline & \multicolumn{2}{|c|}{ VDE (expt) } & \multicolumn{2}{|c|}{ VDE (theor) } & \multirow[b]{2}{*}{$\begin{array}{c}\text { AEA } \\
\text { (expt) }\end{array}$} & \multirow[b]{2}{*}{$\begin{array}{c}\text { AEA } \\
\text { (theor) }\end{array}$} & \multirow[b]{2}{*}{$\begin{array}{l}\text { H-L gap } \\
\text { (theor) }\end{array}$} \\
\hline & first & second & first & second & & & \\
\hline $\mathrm{VNa}_{6}^{-}$ & 1.00 & 1.52 & 0.96 & 1.56 & $\sim 0.9$ & 0.94 & 0.24 \\
\hline isomer & & & 1.26 & 1.28 & & & \\
\hline $\mathrm{VNa}_{7}^{-}$ & 1.00 & 1.23 & 0.99 & 1.24 & $\sim 0.9$ & 0.96 & 0.35 \\
\hline $\mathrm{VNa}_{8}{ }^{-}$ & 0.94 & 1.70 & 0.82 & 1.71 & $\sim 0.8$ & 0.81 & 0.42 \\
\hline $\mathrm{VNa}_{9}{ }^{-}$ & 0.95 & & 0.88 & 0.94 & $\sim 0.8$ & 0.84 & 0.23 \\
\hline $\mathrm{V} 2 \mathrm{Na}_{6}{ }^{-}$ & 1.20 & 1.40 & 1.13 & 1.31 & $\sim 1.0$ & 1.08 & 0.31 \\
\hline $\mathrm{V} 2 \mathrm{Na}_{13}{ }^{-}$ & 1.28 & & 1.25 & & $\sim 1.2$ & 1.24 & 0.30 \\
\hline $\mathrm{V} 2 \mathrm{Na}_{14}{ }^{-}$ & 1.40 & & 1.36 & 1.37 & $\sim 1.3$ & 1.30 & 0.27 \\
\hline $\mathrm{V} 2 \mathrm{Na}_{15}{ }^{-}$ & 1.40 & & 1.35 & 1.37 & $\sim 1.3$ & 1.35 & 0.44 \\
\hline $\mathrm{V} 2 \mathrm{Na}_{16}{ }^{-}$ & 1.50 & 1.60 & 1.38 & 1.49 & $\sim 1.3$ & 1.26 & 0.33 \\
\hline isomer & & & 1.37 & 1.46 & & & \\
\hline
\end{tabular}

For $\mathrm{VNa}_{6}{ }^{-}$, we also show the corresponding transition energies for the isomer. Note that the transition energies for the isomer are significantly different from those for the ground state, indicating that the isomer is not seen under our experimental conditions. This comparison is significant as it shows the sensitivity of the negative ion photoelectron spectra to different geometries. The close agreement between the calculated ground states and the experimental VDE values shows that the calculated ground states are indeed seen in experiments. The case of $\mathrm{VNa}_{8}{ }^{-}$is particularly important as $\mathrm{VNa}_{8}$ was previously identified as a magnetic superatom. ${ }^{16}$ In addition to the calculation of the vertical transitions for $\mathrm{VNa}_{x}{ }^{-}$ with $x=6-9$, we calculated the absorption spectra of the neutral clusters using the TDDFT approximation to gain insight into the photoelectron spectra. As the photoelectron spectrum corresponds to a transition from the ground state of the anion to the various excited states of the neutral, the shape of the spectrum corresponds to the absorption spectrum of the neutral cluster. For a more quantitative comparison, we positioned the calculated absorption spectra by the calculated peak position for the first peak and compared it with experiment. The calculated spectrum is shown by the red curve in Figure 2 and 3. The close agreement between the calculated and the experimental spectra suggests that $\mathrm{VNa}_{8}{ }^{-}$ indeed has a ground-state multiplicity of 7 with a spin magnetic moment of $6.0 \mu_{\mathrm{B}}$, while the neutral $\mathrm{VNa}_{8}$ has a ground state with a multiplicity of 6 or a spin magnetic moment of $5.0 \mu_{\mathrm{B}}$. $\mathrm{VNa}_{8}$ also has the lowest AEA value, consistent with a closed electronic shell nature. All this shows that it is indeed a magnetic superatoms.

In order to investigate the energetic stability of the anionic $\mathrm{VNa}_{x}{ }^{-}$clusters we calculated the energy required to remove a $\mathrm{Na}$ to generate the corresponding $\mathrm{VNa}_{x-1}{ }^{-}$species:

$$
\Delta E=E\left(\mathrm{VNa}_{x}{ }^{-}\right)-E\left(\mathrm{VNa}_{x-1}{ }^{-}\right)-E(\mathrm{Na})
$$

where $E\left(\mathrm{VNa}_{x}{ }^{-}\right), E\left(\mathrm{VNa}_{x-1}{ }^{-}\right)$, and $E(\mathrm{Na})$ are respectively the total energies of the $\mathrm{VNa}_{n}{ }^{-}$and $\mathrm{VNa}_{\mathrm{n}-1}{ }^{-}$clusters, and of a Na atom. A large $\Delta E$ is characteristic of a stable cluster. As shown in Figure $\mathrm{S} 3, \mathrm{VNa}_{8}{ }^{-}$presents the largest $\Delta E$ among the series accounting for its stability.

The other interesting cluster is $\mathrm{VNa}_{7}^{-}$as it has the highest intensity in the mass spectrum of the $\mathrm{VNa}_{x}{ }^{-}$series. As shown in Table 1, it also has the highest calculated AEA value, i.e., 0.96 $\mathrm{eV}$. This cluster also has a gap of $0.35 \mathrm{eV}$ between the highest occupied molecular orbital (HOMO) and the lowest unoccupied molecular orbital (LUMO). This gap is large although smaller than in covalently bonded $\mathrm{C}_{60}$, where the H-L gap is $1.7 \mathrm{eV}^{35}$ Combined with its high electron affinity, this suggests that the anionic species is highly stable, which is consistent with the result that it is the strongest peak in the mass spectrum. As the cluster has the same valence count as $\mathrm{VNa}_{8}$, we proceeded to examine the nature of electronic states contributing to its magnetic character.

Figure 5 shows the one-electron levels and the shape of the associated electronic orbitals in anionic $\mathrm{VNa}_{7}{ }^{-}, \mathrm{VNa}_{8}{ }^{-}$, and neutral $\mathrm{VNa}_{8}$ and $\mathrm{VNa}_{9}$ clusters. The electronic orbitals have been assigned approximate orbital character through inspection of the nodes. $\mathrm{VNa}_{7}^{-}$has 13 valence electrons, and Figure 5 shows that its valence configuration corresponds to a $1 \mathrm{~S}^{2} 1 \mathrm{P}^{6} 1 \mathrm{D}_{\alpha}^{5}$ superatomic configuration like in a $\mathrm{Mn}$ atom. The $S$ - and P-states are fairly diffuse over the entire cluster while the $\mathrm{D}$-states have a large amplitude on $\mathrm{V}$ sites indicating a strong hybridization with $\mathrm{V}$ 3d-states. The same orbital sequence is seen in neutral $\mathrm{VNa}_{8}$. The strong hybridization is the origin of the large exchange splitting of around $1.1 \mathrm{eV}$ in $\mathrm{VNa}_{7}{ }^{-}$or $1.02 \mathrm{eV}$ in $\mathrm{VNa}_{8}$. The large splitting coupled with filled majority d-subshell results in a spin magnetic moment of $5 \mu_{\mathrm{B}}$. Because of the similar parity, the atomic exchange splitting is also transmitted to $\mathrm{S}$-states that lead to a spin magnetic moment of $6 \mu_{\mathrm{B}}$ in $\mathrm{VNa}_{8}{ }^{-}$or $\mathrm{VNa}_{9}$ leading to a superatomic configuration of $1 S^{2} 1 \mathrm{P}^{6} 1 \mathrm{D}_{\alpha}{ }^{5} 2 \mathrm{~S}_{\alpha}{ }^{1}$. This filling is reminiscent of the atomic situation in a $\mathrm{Cr}$ atom. In fact $\mathrm{VNa}_{8}{ }^{-}$has a higher $\mathrm{H}$ $\mathrm{L}$ gap of $0.42 \mathrm{eV}$ than $\mathrm{VNa}_{7}{ }^{-}$and the largest $\Delta E$ and hence can be regarded as a stable species as seen in Figure S3. One can therefore regard $\mathrm{VNa}_{8}$ and $\mathrm{VNa}_{8}{ }^{-}$as magnetic superatoms resembling subshell filling in $\mathrm{Mn}$ and $\mathrm{Cr}$ atoms, respectively. With magnetic electrons localized on the $\mathrm{V}$ site, the clusters will have negligible magnetic anisotropy making them paramagnetic like ordinary atoms. They can be classified as magnetic superatoms.

Our studies on $\mathrm{V}_{2} \mathrm{Na}_{y}{ }^{-}$clusters show that the ground states correspond to the configurations where the $\mathrm{V}$ atoms combine to form a $\mathrm{V}_{2}$ dimer. As the dimer is surrounded by $\mathrm{Na}$ atoms, the ground states correspond to the lowest spin multiplicity (singlet for even electron systems and doublet for odd electron systems). The theoretical ground states are again in agreement with experimental negative ion photoelectron spectrum as confirmed by the peak positions in the photoelectron spectrum. This comparison is given in Table 1. It shows the positions of 

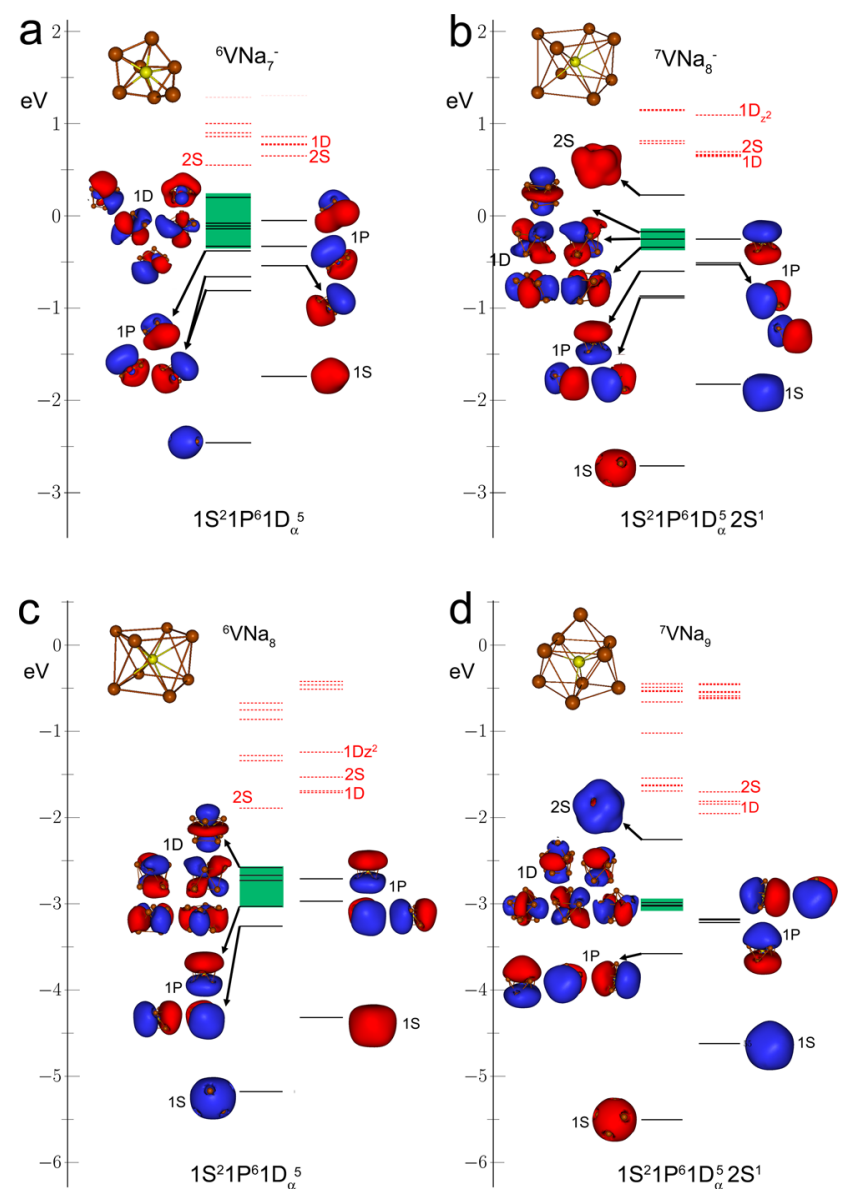

Figure 5. One-electron energy levels and molecular orbital charge density isosurfaces (isovalue $0.001 \mathrm{C} \mathrm{m}^{-3}$ ) in the $\mathrm{VNa}_{7}^{-}$(a), $\mathrm{VNa}_{8}^{-}$ (b), $\mathrm{VNa}_{8}$ (c), and $\mathrm{VNa}_{9}$ (d) clusters. The majority and minority levels are shown. Continuous lines correspond to the filled levels and the dotted lines correspond to the unfilled states. The angular momentum of the delocalized molecular orbitals is indicated. The 1D majority levels are marked with a green square.

the first and second peak in experiment and the corresponding theoretical values for the cases where the experimental spectra can be obtained. The close agreement in all the cases indicates that the theoretical ground states are seen in experiments.

\section{CONCLUSIONS}

The identification of $\mathrm{VNa}_{x}$ magnetic superatoms is particularly interesting if such clusters could be supported on substrates and used to generate spin polarized currents. In an earlier study, we had shown that a dimer composed of two $\mathrm{VCs}_{8}$ clusters could lead to spin polarized currents with over $90 \%$ spin polarization. ${ }^{36}$ The stumbling blocks to realize these potentials is the synthesis of such superatoms. The present work opens the door to these important applications as it demonstrates that it is possible to generate magnetic superatoms in carefully planned experiments. A comparison of the experimental photoelectron spectrum with theoretical transitions also enables a determination of the spin magnetic moment of the cluster which extends our earlier work showing that $\mathrm{VNa}_{7}{ }^{-}, \mathrm{VNa}_{8}, \mathrm{VNa}_{8}{ }^{-}$, and $\mathrm{VNa}_{9}$ are all stable magnetic superatoms. The filled $\mathrm{D}_{\alpha}$ or $\mathrm{D}_{\alpha} \mathrm{S}_{\alpha}$ subshells lead to high spin magnetic moments of 5 and 6 $\mu_{\mathrm{B}}$, respectively. The ability to synthesize such clusters in molecular beams opens a new era of making such devices under controlled situations, and we hope that the present findings will stimulate further efforts to assemble such motifs.

\section{ASSOCIATED CONTENT}

\section{Supporting Information}

Photoelectron spectra at $355 \mathrm{~nm}$ for V2Nax clusters $(n=6$, 13-16) and theoretical detachment energies (VDE); groundstate geometries of neutral and anionic V2Nax $(n=4-16)$ clusters along with their total energies. This material is available free of charge via the Internet at http://pubs.acs.org.

\section{AUTHOR INFORMATION}

\section{Corresponding Author}

snkhanna@vcu.edu;kbowen@jhu.edu

Notes

The authors declare no competing financial interest.

\section{ACKNOWLEDGMENTS}

J.U.R. and S.N.K. gratefully acknowledge support from the U.S. Department of Energy through grant DE-FG02-11ER16213. K.H.B. gratefully acknowledge support from the National Science Foundation through grant No. 1111693.

\section{REFERENCES}

(1) Knight, W. D.; Clemenger, K.; de Heer, W. A.; Saunders, W. A.; Chou, M. Y.; Cohen, M. L. Phys. Rev. Lett. 1984, 52, 2141.

(2) Leuchtner, R. E.; Harms, A. C.; Castleman, A. W., Jr. J. Chem. Phys. 1989, 91, 2753-2754.

(3) Brack, M. Rev. Mod. Phys. 1993, 65, 677-732.

(4) Khanna, S. N.; Jena, P. Phys. Rev. B 1995, 51, 13705-13716.

(5) Ekardt, W. Z. Phys. B Cond. Mat. 1997, 103, 305-312.

(6) Jug, K.; Zimmermann, B.; Köster, A. M. Int. J. Quantum Chem. 2002, 90, 594-602.

(7) Kumar, V.; Kawazoe, Y. Appl. Phys. Lett. 2002, 80, 859-861.

(8) Neukermans, S.; Janssen, E.; Chen, Z. F.; Silverans, R. E.; Schleyer, P. v. R.; Lievens, P. Phys. Rev. Lett. 2004, 92, 163401.

(9) Bergeron, D. E.; Castleman, A. W., Jr.; Morisato, T.; Khanna, S. N. Science 2004, 304, 84-87.

(10) Bergeron, D. E.; Roach, P. J.; Castleman, A. W., Jr.; Jones, N. O.; Khanna, S. N. Science 2005, 307, 231-235.

(11) Janssens, E.; Neukermans, S.; Lievens, P. Curr. Opin. Solid State Mater. Sci. 2004, 8, 185-193.

(12) Reveles, J. U.; Khanna, S. N.; Roach, P. J.; Castleman, A. W., Jr. Proc. Natl. Acad. Sci. U.S.A. 2006, 103, 18405-18410.

(13) Jadzinsky, P. D.; Calero, G.; Ackerson, C. J.; Bushnell, D. A.; Kornberg, R. D. Science 2007, 318, 430-433.

(14) Walter, M.; Akola, J.; Lopez-Acevedo, O.; Jadzinsky, P. D.; Calero, G.; Ackerson, C. J.; Whetten, R. L.; Gronbeck, H.; Hakkinen, H. Proc. Natl. Acad. Sci. U.S.A. 2008, 105, 9157-9162.

(15) Hartig, J.; Stösser, A.; Hauser, P.; Schnöckel, H. Angew. Chem., Int Ed. 2007, 46, 1658-1662.

(16) Castleman, A. W., Jr.; Khanna, S. N. J. Phys. Chem. C 2009, 113, 2664-2675.

(17) Reveles, J. U.; Clayborne, P. A.; Reber, A. C.; Khanna, S. N.; Pradhan, K.; Sen, S.; Pederson, M. R. Nat. Chem. 2009, 1, 310-315.

(18) Khanna, S. N.; Rao, B. K.; Jena, P.; Martins, J. L. In Physics and Chemistry of Small Clusters; Jena, P., Rao, B. K., Khanna, S. N., Eds.; Plenum Press: New York, 1987; pp 435-438.

(19) Cox, A. J.; Louderback, J. G.; Apsel, S. E.; Bloomfield, L. A. Phys. Rev. B 1994, 49, 12295-12298.

(20) Khanna, S. N.; Linderoth, S. Phys. Rev. Lett. 1991, 67, 742-745.

(21) Engelking, P. C.; Lineberger, W. C. Phys. Rev. A 1979, 19, 149.

(22) Sobhy, M. A.; Reveles, J. U.; Gupta, U.; Khanna, S. N.; Castleman, A. W., Jr. J. Chem. Phys. 2009, 130, 054304.

(23) Gutsev, G. L.; Khanna, S. N.; Jena, P. Phys. Rev. B 2000, 62, $1604-1606$ 
(24) Stokes, S. T.; Li, X.; Grubisic, A.; Ko, Y. J.; Bowen, K. H. J. Chem. Phys. 2007, 127, 084321.

(25) Thomas, O. C.; Zheng, W. J.; Bowen, K. H. J. Chem. Phys. 2001, $114,5514$.

(26) Bilodeau, R. C.; Scheer, M.; Haugen, H. K. J. Phys. B 1998, 31, 3885.

(27) Li, X.; Grubisic, A.; Stokes, S. T.; Cordes, J.; Gantefoer, G. F.; Bowen, K. H.; Kiran, B.; Willis, M.; Jena, P.; Burgert, R.; Schnockel, H. Science 2007, 315, 356.

(28) Geudtner, G.; Calaminici, P.; Carmona-Espindola, J., M.; del Campo, J. M.; Dominguez-Soria, V. D.; Flores-Moreno, R.; Gamboa, G. U.; Goursot, A.; Koster, A. M.; Reveles, J. U.; Tzonka, M.; VasquezPerez, J. M.; Vela, A.; Zuninga-Gutierrez, B.; Salahub, D. R. WIREs Comput. Mol. Sci. 2012, 2, 548-555.

(29) Köster, A. M.; Geudtner, G.; Calaminici, P.; Casida, M. E.; Dominguez, V. D.; Flores-Moreno, R.; Goursot, A.; Heine, T.; Ipatov, A.; Janetzko, F.; Martin del Campo, J.; Reveles, J. U.; Vela, A.; Zuniga, B.; Salahub, D. R. deMon; Cinvestav: Mexico, 2011; http://www. deMon-software.com.

(30) Perdew, J. P.; Burke, K.; Enzerhof, M. Phys. Rev. Lett. 1996, 77, 3865-3868.

(31) Dunlap, B. I.; Connolly, J. W. D.; Sabin, J. R. J. Chem. Phys. 1979, 71, 3396.

(32) Calaminici, P.; Janetzko, F.; Köster, A. M.; Mejia-Olvera, R.; Zuniga-Gutierrez, B. J. Chem. Phys. 2007, 126, 044108.

(33) Reveles, J. U.; Köster, A. M. J. Comput. Chem. 2004, 25, 1109.

(34) Ipatov, A.; Fouqueau, A.; del Valle, C. P.; Cordova, F.; Casida, M. E.; Koster, A. M.; Vela, A.; Jamorski, C. J. J. Mol. Struct.: THEOCHEM 2006, 762, 179.

(35) Tamai, A.; Seitsonen, A. P.; Baumberger, F.; Hangsberger, M.; Shen, Z.-X.; Greber, T.; Osterwalder, J. Phys. Rev. B 2008, 77, 0751341-075134-8.

(36) He, H.; Pandey, R.; Reveles, J. U.; Khanna, S. N.; Karna, S. S. Appl. Phys. Lett. 2009, 95, 192104. 